\title{
Tamanhos de unidades experimentais básicas e de parcelas em tremoço branco
}

\author{
Basic experimental units and plot sizes in white lupine
}

\author{
Alberto Cargnelutti Filho ${ }^{I}$ Bruna Mendonça Alves ${ }^{I I}$ \\ Marcos Toebe ${ }^{\text {III }}$ Giovani Facco ${ }^{\text {II }}$
}

\section{RESUMO}

O objetivo deste trabalho foi verificar a influência do tamanho da unidade experimental básica (UEB) na estimativa do tamanho ótimo de parcela, para a avaliação da massa de matéria verde de tremoço branco (Lupinus albus L.). Avaliou-se a massa de matéria verde aos 123, 137 e 150 dias após a semeadura. Em cada época, foram avaliadas $432 U E B$ de $1 \mathrm{~m} \times 1 \mathrm{~m}\left(1 \mathrm{~m}^{2}\right)$ e formaram-se 16 planos de UEB com tamanhos entre 1 e $16 \mathrm{~m}^{2}$. Para cada plano de UEB, foi determinado o tamanho ótimo de parcela, pelo método da curvatura máxima do modelo do coeficiente de variação. Em cada época de avaliação, foi calculada a diferença mínima significativa pelo teste de Tukey, em 448 cenários formados pelas combinações de 16 tamanhos ótimos de parcela, i tratamentos $(i=5,10,15$ e 20) e r repetições $(r=3,4,5,6,10,15$ e 20), para $o$ delineamento blocos ao acaso. A estimativa do tamanho ótimo de parcela depende do tamanho da unidade experimental básica. É indicado avaliar a massa de matéria verde em UEB de menor tamanho possivel, para serem usadas na estimação do tamanho ótimo de parcela.

Palavras-chave: Lupinus albus L., planejamento experimental, diferença minima significativa.

\section{ABSTRACT}

The objective of this research was to determine the influence of the basic experimental unit size (BEU) to estimate the optimum plot size, in the evaluation of white lupine (Lupinus albus L.) fresh matter weight. Data from fresh matter weight to 123, 137 and 150 days after sowing were evaluated. In every season $432 \mathrm{BEU} 1 \mathrm{~m} \times 1 \mathrm{~m}\left(1 \mathrm{~m}^{2}\right)$ were evaluated and $16 \mathrm{BEU}$ plans of sizes from 1 to $16 \mathrm{~m}^{2}$ were formed. For each BEU plan, the optimum plot size was determined by the method of maximum curvature of the coefficient of variation model. The least significant difference by
Tukey's test in 448 scenarios formed by the combinations of 16 plot sizes, $i$ treatments $(i=5,10,15$ and 20$)$, and $r$ replicates $(r=3,4$, 5, 6, 10, 15 and 20) for randomized block designs was calculated. Estimate of optimum plot size depends of the basic experimental unit size. It is indicated to evaluate the fresh matter weight in basic experimental units smallest possible size, for use in the estimation of optimum plot size.

Key words: Lupinus albus L., experimental design, least significant difference.

\section{INTRODUÇÃO}

A avaliação da massa de matéria verde de tremoço branco (Lupinus albus L.) em experimentos com essa importante cultura de cobertura de solo, deve ser precisa para que os resultados sejam confiáveis. A definição do tamanho ótimo de parcela é um aspecto importante a ser observado ao planejar o experimento, a fim de minimizar o erro experimental. $\mathrm{O}$ tamanho ótimo de parcela para avaliar a massa verde total, a massa verde de parte aérea e a massa verde de raízes de tremoço branco (Lupinus albus L.), em épocas de avaliação, estudado por CARGNELUTTI FILHO et al. (2015), foi de $7,48 \mathrm{~m}^{2}$.

Experimentos com a cultura de tremoço branco, avaliando a massa verde, foram conduzidos no delineamento experimental de blocos ao acaso, utilizando distintos tamanhos de parcela e número

\footnotetext{
'Departamento de Fitotecnia, Centro de Ciências Rurais (CCR), Universidade Federal de Santa Maria (UFSM), 97105-900, Santa Maria, RS, Brasil. E-mail: alberto.cargnelutti.filho@gmail.com. Autor para correspondência.

"Programa de Pós-graduação em Agronomia, Centro de Ciências Rurais (CCR), Universidade Federal de Santa Maria (UFSM), Santa Maria, RS, Brasil.

IIIFundação Universidade Federal do Pampa (UNIPAMPA), Itaqui, RS, Brasil. 
de repetições, como parcela de $20 \mathrm{~m}^{2}(5,0 \mathrm{~m} \times 4,0 \mathrm{~m})$ e três repetições (POTT et al., 2007), parcela de $37,5 \mathrm{~m}^{2}(7,5 \mathrm{~m} \times 5,0 \mathrm{~m})$ e quatro repetições (PEREIRA et al., 2012) e ainda parcelas de $24 \mathrm{~m}^{2}(6 \mathrm{~m} \times 4 \mathrm{~m})$ e seis repetições (SOUZA \& GUIMARÃES, 2013).

Ensaios de uniformidade (experimentos em branco) são definidos como ensaios sem tratamentos (STORCK et al., 2011), nos quais são realizados os mesmos tratos culturais em toda a área experimental. Os dados coletados em unidades experimentais básicas desses ensaios podem ser utilizados para a determinação do tamanho ótimo de parcela, por distintas metodologias. O método da curvatura máxima do modelo do coeficiente de variação, proposto por PARANAÍBA et al. (2009), é de aplicação simples, principalmente por não necessitar o agrupamento de unidades experimentais básicas adjacentes, e foi aplicado em tremoço branco (CARGNELUTTI FILHO et al., 2015).

Em um ensaio de uniformidade, é recomendado dividir a área experimental em unidades experimentais básicas de menor tamanho possível e compatíveis com a variável mensurada (STORCK et al., 2011). Porém, um aspecto importante e ainda pouco estudado é a influência do tamanho da unidade experimental básica na estimativa do tamanho ótimo de parcela. Na cultura de batata (Solanum tuberosum L.), o efeito do tamanho da unidade experimental básica $(1,2,3,4,6,8$ e 12 covas) sobre o tamanho ótimo de parcela, estimado pelo método da curvatura máxima modificada (MEIER \& LESSMAN, 1971), foi estudado por OLIVEIRA et al. (2005). Os autores concluíram que o tamanho da unidade experimental básica afeta a estimativa do tamanho ótimo de parcela sem alterar a precisão experimental, independentemente do número de tratamentos, mantendo fixa a área total do experimento.

Investigações sobre as estimativas de tamanho ótimo de parcela, obtidas pelo método da curvatura máxima do modelo do coeficiente de variação (PARANAÍBA et al., 2009), a partir de distintos tamanhos de unidades experimentais básicas com a cultura de tremoço branco, não foram encontradas na literatura. Essas investigações podem ser realizadas a partir de dados de ensaios de uniformidade e podem fornecer informações úteis, pois planejamentos experimentais, em cenários formados por combinações de tamanhos de parcela e de números de tratamentos e de repetições, podem gerar distintos níveis de precisão experimental. Assim, o objetivo deste trabalho foi verificar a influência do tamanho da unidade experimental básica na estimativa do tamanho ótimo de parcela, para a avaliação da massa de matéria verde de tremoço branco (Lupinus albus L.).

\section{MATERIAL E MÉTODOS}

Foi realizado um ensaio de uniformidade (experimento em branco) com a cultura de tremoço branco (Lupinus albus L.), em área experimental de $50 \mathrm{~m} \times 50 \mathrm{~m}$ do Departamento de Fitotecnia, da Universidade Federal de Santa Maria, Santa Maria, Estado do Rio Grande do Sul. Pela classificação de Köppen, o clima da região é do tipo Cfa, subtropical úmido, com verões quentes e sem estação seca definida (HELDWEIN et al., 2009). O solo é classificado como Argissolo Vermelho distrófico arênico (SANTOS et al., 2006). A semeadura foi realizada a lanço, no dia 13 de junho de 2011. A adubação de base foi de $30 \mathrm{~kg}$ $\mathrm{ha}^{-1}$ de $\mathrm{N}, 120 \mathrm{~kg} \mathrm{ha}^{-1}$ de $\mathrm{P}_{2} \mathrm{O}_{5}$ e $120 \mathrm{~kg} \mathrm{ha}^{-1}$ de $\mathrm{K}_{2} \mathrm{O}$. Os tratos culturais, no ensaio de uniformidade, foram os mesmos em toda a área experimental, conforme preconizado por STORCK et al. (2011).

Na parte central do ensaio de uniformidade, foram marcadas três áreas de $36 \mathrm{~m} \times 12 \mathrm{~m}\left(432 \mathrm{~m}^{2}\right)$. Cada área foi dividida em 432 unidades experimentais básicas (UEB) de $1 \mathrm{~m} \times 1 \mathrm{~m}\left(1 \mathrm{~m}^{2}\right)$ e formou uma matriz de 36 linhas e 12 colunas. $\mathrm{Na}$ área um, aos 123 dias após a semeadura (DAS, primeira época de avaliação), na área dois, aos 137DAS (segunda época de avaliação) e, na área três, aos 150DAS (terceira época de avaliação), em cada UEB, as plantas foram arrancadas e cortadas para separação de raízes e parte aérea. As raízes não foram utilizadas nesse estudo. A parte aérea foi pesada e obteve-se a massa de matéria verde, em gramas.

Em cada época de avaliação, com os dados de massa de matéria verde das 432UEB, foram formados 16 planos de UEB, com tamanhos $\mathrm{X}=\mathrm{X}_{\mathrm{L}} \times \mathrm{X}_{\mathrm{C}}(\mathrm{X}=1,2$, $3,4,6,8,9,12,16 \mathrm{~m}^{2}$ ) e formas $\mathrm{X}_{\mathrm{L}} \times \mathrm{X}_{\mathrm{C}}$ (quadrada $\mathrm{e}$ retangular) (Tabela 1). As siglas $\mathrm{X}_{\mathrm{L}}, \mathrm{X}_{\mathrm{C}}$ e X significam, respectivamente, número de UEB adjacentes à linha, número de UEB adjacentes à coluna e tamanho de UEB, em número de UEB ou em metros quadrados. Assim, os 16 planos de UEB foram formados entre $1 \times 1\left(1 \mathrm{UEB}=1 \mathrm{~m}^{2}\right)$ e $4 \times 4\left(16 \mathrm{UEB}=16 \mathrm{~m}^{2}\right)$ e, para a sua composição, foram somados os valores de massa de matéria verde de $\mathrm{X}_{\mathrm{L}}$ UEB adjacentes à linha e de $\mathrm{X}_{\mathrm{C}}$ UEB adjacentes à coluna.

Para cada plano de UEB, com os dados de massa de matéria verde, determinou-se o coeficiente de autocorrelação espacial de primeira ordem $(\rho)$, a variância $\left(\mathrm{s}^{2}\right)$, o desvio padrão (s), a média $(\mathrm{m})$ e o coeficiente de variação do ensaio $(\mathrm{CV}=100 \mathrm{~s} / \mathrm{m}$, em \%). A estimativa de $\rho$ foi obtida no sentido das 
Tabela 1 - Planos de unidades experimentais básicas (UEB) com tamanhos $X=X_{L} \times X_{C}$, em UEB e em $m^{2}$, e respectivas estimativas de coeficiente de autocorrelação espacial de primeira ordem $(\rho)$, variância $\left(\mathrm{s}^{2}\right)$, desvio padrão $(\mathrm{s})$, média $(\mathrm{m})$, coeficiente de variação do ensaio (CV, em \%), tamanho ótimo de parcela (Xo, em UEB) e tamanho ótimo de parcela (Xo, em $\left.\mathrm{m}^{2}\right)$, para a massa de matéria verde de tremoço branco (Lupinus albus L.), em gramas $\mathrm{m}^{-2}$, avaliada aos 123, 137 e 150 dias após a semeadura (DAS), em ensaio de uniformidade com 1.296 UEB de $1 \mathrm{~m} \times 1 \mathrm{~m}\left(1 \mathrm{~m}^{2}\right)$.

\begin{tabular}{|c|c|c|c|c|c|c|c|c|c|c|c|c|c|}
\hline DAS & Plano & $\mathrm{X}_{\mathrm{L}}$ & $\mathrm{X}_{\mathrm{C}}$ & X (UEB) & $\mathrm{X}\left(\mathrm{m}^{2}\right)$ & $\mathrm{n}$ & $\rho$ & $s^{2}$ & $\mathrm{~s}$ & $\mathrm{~m}$ & $\mathrm{CV}(\%)$ & Xo (UEB) & $\mathrm{Xo}\left(\mathrm{m}^{2}\right)$ \\
\hline 123 & 1 & 1 & 1 & 1 & 1 & 432 & 0,38 & $213.700,50$ & 462,28 & $1.057,34$ & 43,72 & 6,90 & 6,90 \\
\hline 123 & 2 & 1 & 2 & 2 & 2 & 216 & 0,36 & $569.464,64$ & 754,63 & $2.114,69$ & 35,69 & 6,04 & 12,09 \\
\hline 123 & 3 & 2 & 1 & 2 & 2 & 216 & 0,52 & $567.944,75$ & 753,62 & $2.114,69$ & 35,64 & 5,70 & 11,41 \\
\hline 123 & 4 & 1 & 3 & 3 & 3 & 144 & 0,34 & $995.759,19$ & 997,88 & $3.172,03$ & 31,46 & 5,59 & 16,77 \\
\hline 123 & 5 & 3 & 1 & 3 & 3 & 144 & 0,58 & $1.033 .774,06$ & $1.016,75$ & $3.172,03$ & 32,05 & 5,14 & 15,41 \\
\hline 123 & 6 & 1 & 4 & 4 & 4 & 108 & 0,32 & $1.572 .131,15$ & $1.253,85$ & $4.229,38$ & 29,65 & 5,40 & 21,60 \\
\hline 123 & 7 & 2 & 2 & 4 & 4 & 108 & 0,47 & $1.626 .819,66$ & $1.275,47$ & $4.229,38$ & 30,16 & 5,21 & 20,85 \\
\hline 123 & 8 & 4 & 1 & 4 & 4 & 108 & 0,59 & $1.679 .460,72$ & $1.295,94$ & $4.229,38$ & 30,64 & 4,97 & 19,87 \\
\hline 123 & 9 & 2 & 3 & 6 & 6 & 72 & 0,39 & $3.043 .158,60$ & $1.744,47$ & $6.344,07$ & 27,50 & 5,04 & 30,27 \\
\hline 123 & 10 & 3 & 2 & 6 & 6 & 72 & 0,51 & $3.122 .036,57$ & $1.766,93$ & $6.344,07$ & 27,85 & 4,85 & 29,10 \\
\hline 123 & 11 & 2 & 4 & 8 & 8 & 54 & 0,46 & $4.708 .773,39$ & $2.169,97$ & $8.458,76$ & 25,65 & 4,70 & 37,61 \\
\hline 123 & 12 & 4 & 2 & 8 & 8 & 54 & 0,49 & $5.065 .185,02$ & $2.250,60$ & $8.458,76$ & 26,61 & 4,75 & 38,00 \\
\hline 123 & 13 & 3 & 3 & 9 & 9 & 48 & 0,40 & $6.154 .279,93$ & $2.480,78$ & $9.516,10$ & 26,07 & 4,84 & 43,60 \\
\hline 123 & 14 & 3 & 4 & 12 & 12 & 36 & 0,49 & $9.166 .974,75$ & $3.027,70$ & $12.688,14$ & 23,86 & 4,43 & 53,15 \\
\hline 123 & 15 & 4 & 3 & 12 & 12 & 36 & 0,42 & $9.766 .001,89$ & $3.125,06$ & $12.688,14$ & 24,63 & 4,65 & 55,75 \\
\hline 123 & 16 & 4 & 4 & 16 & 16 & 27 & 0,52 & $15.363 .519,11$ & $3.919,63$ & $16.917,52$ & 23,17 & 4,28 & 68,53 \\
\hline 137 & 1 & 1 & 1 & 1 & 1 & 432 & 0,29 & $473.148,52$ & 687,86 & $2.069,91$ & 33,23 & 5,87 & 5,87 \\
\hline 137 & 2 & 1 & 2 & 2 & 2 & 216 & 0,34 & $1.207 .766,95$ & $1.098,98$ & $4.139,81$ & 26,55 & 4,99 & 9,98 \\
\hline 137 & 3 & 2 & 1 & 2 & 2 & 216 & 0,43 & $1.089 .618,43$ & $1.043,85$ & $4.139,81$ & 25,21 & 4,70 & 9,40 \\
\hline 137 & 4 & 1 & 3 & 3 & 3 & 144 & 0,39 & $2.082 .276,96$ & $1.443,01$ & $6.209,72$ & 23,24 & 4,51 & 13,52 \\
\hline 137 & 5 & 3 & 1 & 3 & 3 & 144 & 0,49 & $2.107 .079,76$ & $1.451,58$ & $6.209,72$ & 23,38 & 4,36 & 13,09 \\
\hline 137 & 6 & 1 & 4 & 4 & 4 & 108 & 0,24 & $3.178 .069,68$ & $1.782,71$ & $8.279,62$ & 21,53 & 4,44 & 17,76 \\
\hline 137 & 7 & 2 & 2 & 4 & 4 & 108 & 0,42 & $3.079 .890,67$ & $1.754,96$ & $8.279,62$ & 21,20 & 4,19 & 16,77 \\
\hline 137 & 8 & 4 & 1 & 4 & 4 & 108 & 0,59 & $3.284 .658,05$ & $1.812,36$ & $8.279,62$ & 21,89 & 3,96 & 15,85 \\
\hline 137 & 9 & 2 & 3 & 6 & 6 & 72 & 0,46 & $5.799 .791,49$ & $2.408,28$ & $12.419,43$ & 19,39 & 3,89 & 23,35 \\
\hline 137 & 10 & 3 & 2 & 6 & 6 & 72 & 0,48 & $6.265 .999,23$ & $2.503,20$ & $12.419,43$ & 20,16 & 3,97 & 23,84 \\
\hline 137 & 11 & 2 & 4 & 8 & 8 & 54 & 0,43 & $7.919 .986,22$ & $2.814,25$ & $16.559,24$ & 17,00 & 3,61 & 28,88 \\
\hline 137 & 12 & 4 & 2 & 8 & 8 & 54 & 0,44 & $10.511 .611,66$ & $3.242,16$ & $16.559,24$ & 19,58 & 3,95 & 31,63 \\
\hline 137 & 13 & 3 & 3 & 9 & 9 & 48 & 0,48 & $12.358 .985,83$ & $3.515,53$ & $18.629,15$ & 18,87 & 3,80 & 34,16 \\
\hline 137 & 14 & 3 & 4 & 12 & 12 & 36 & 0,39 & $17.453 .186,98$ & $4.177,70$ & $24.838,86$ & 16,82 & 3,63 & 43,60 \\
\hline 137 & 15 & 4 & 3 & 12 & 12 & 36 & 0,45 & $20.351 .379,67$ & $4.511,25$ & $24.838,86$ & 18,16 & 3,75 & 44,98 \\
\hline 137 & 16 & 4 & 4 & 16 & 16 & 27 & 0,35 & $29.340 .615,49$ & $5.416,70$ & $33.118,48$ & 16,36 & 3,61 & 57,73 \\
\hline 150 & 1 & 1 & 1 & 1 & 1 & 432 & 0,41 & $1.197 .382,21$ & $1.094,25$ & $3.053,18$ & 35,84 & 5,98 & 5,98 \\
\hline 150 & 2 & 1 & 2 & 2 & 2 & 216 & 0,35 & $3.397 .719,25$ & $1.843,29$ & $6.106,36$ & 30,19 & 5,42 & 10,85 \\
\hline 150 & 3 & 2 & 1 & 2 & 2 & 216 & 0,55 & $3.305 .066,98$ & $1.817,98$ & $6.106,36$ & 29,77 & 4,99 & 9,98 \\
\hline 150 & 4 & 1 & 3 & 3 & 3 & 144 & 0,30 & $6.215 .021,59$ & $2.492,99$ & $9.159,53$ & 27,22 & 5,13 & 15,40 \\
\hline 150 & 5 & 3 & 1 & 3 & 3 & 144 & 0,60 & $6.114 .829,09$ & $2.472,82$ & $9.159,53$ & 27,00 & 4,52 & 13,57 \\
\hline 150 & 6 & 1 & 4 & 4 & 4 & 108 & 0,39 & $8.679 .612,19$ & $2.946,12$ & $12.212,71$ & 24,12 & 4,62 & 18,50 \\
\hline 150 & 7 & 2 & 2 & 4 & 4 & 108 & 0,37 & $10.238 .884,51$ & $3.199,83$ & $12.212,71$ & 26,20 & 4,91 & 19,62 \\
\hline 150 & 8 & 4 & 1 & 4 & 4 & 108 & 0,62 & $10.408 .520,21$ & $3.226,22$ & $12.212,71$ & 26,42 & 4,41 & 17,63 \\
\hline 150 & 9 & 2 & 3 & 6 & 6 & 72 & 0,34 & $19.002 .040,80$ & $4.359,13$ & $18.319,07$ & 23,80 & 4,64 & 27,86 \\
\hline 150 & 10 & 3 & 2 & 6 & 6 & 72 & 0,40 & $19.752 .979,70$ & $4.444,43$ & $18.319,07$ & 24,26 & 4,63 & 27,78 \\
\hline 150 & 11 & 2 & 4 & 8 & 8 & 54 & 0,48 & $25.756 .131,15$ & $5.075,05$ & $24.425,43$ & 20,78 & 4,04 & 32,35 \\
\hline 150 & 12 & 4 & 2 & 8 & 8 & 54 & 0,40 & $33.837 .506,51$ & $5.817,00$ & $24.425,43$ & 23,82 & 4,57 & 36,54 \\
\hline 150 & 13 & 3 & 3 & 9 & 9 & 48 & 0,36 & $36.082 .636,46$ & $6.006,88$ & $27.478,60$ & 21,86 & 4,36 & 39,27 \\
\hline 150 & 14 & 3 & 4 & 12 & 12 & 36 & 0,48 & $51.550 .718,87$ & $7.179,88$ & $36.638,14$ & 19,60 & 3,90 & 46,77 \\
\hline 150 & 15 & 4 & 3 & 12 & 12 & 36 & 0,37 & $62.574 .570,12$ & $7.910,41$ & $36.638,14$ & 21,59 & 4,32 & 51,86 \\
\hline 150 & 16 & 4 & 4 & 16 & 16 & 27 & 0,49 & $88.352 .986,44$ & $9.399,63$ & $48.850,85$ & 19,24 & 3,83 & 61,28 \\
\hline
\end{tabular}

$\mathrm{X}_{\mathrm{L}}$ : UEB adjacentes à linha; $\mathrm{X}_{\mathrm{C}}$ : UEB adjacentes à coluna; $\mathrm{n}$, número de UEB com $\mathrm{X}$ UEB de tamanho $(\mathrm{n}=432 / \mathrm{X})$.

linhas, conforme metodologia de PARANAÍBA et al. (2009). Com base no método da curvatura máxima do modelo do coeficiente de variação, proposto por PARANAÍBA et al. (2009), foi determinado o tamanho ótimo de parcela (Xo), em UEB, por
$\mathrm{Xo}=\left[10 \sqrt[3]{2\left(1-\rho^{2}\right) \mathrm{s}^{2} \mathrm{~m}}\right) / \mathrm{m}$. Determinou-se o tamanho ótimo de parcela (Xo), em $\mathrm{m}^{2}$, pelo produto de Xo, em UEB, pela área da UEB, em $\mathrm{m}^{2}$. Após, foram ajustados modelos do tipo linear e potência, a partir dos 16 tamanhos de UEB (X, em UEB) (variável 
independente) e das estimativas do desvio padrão (s), média $(\mathrm{m})$, coeficiente de variação do ensaio $(\mathrm{CV}$, em \%), tamanho ótimo de parcela (Xo, em UEB) e tamanho ótimo de parcela $\left(\mathrm{Xo}, \mathrm{em} \mathrm{m}^{2}\right)$ (variáveis dependentes), e determinados os coeficientes de determinação $\left(\mathrm{R}^{2}\right)$. Esses ajustes foram realizados no aplicativo Microsoft Office Excel ${ }^{\circledR}$, por meio do método dos mínimos quadrados.

Para avaliar a precisão experimental em combinações de tamanhos de parcela, números de tratamentos e de repetições no delineamento blocos ao acaso, assumiu-se cada plano de UEB (16 planos) de tamanho $\mathrm{X}=\mathrm{X}_{\mathrm{L}} \times \mathrm{X}_{\mathrm{C}}(\mathrm{X}=1,2,3,4,6,8,9,12$, $16 \mathrm{~m}^{2}$ ) e formato $\mathrm{X}_{\mathrm{L}} \times \mathrm{X}_{\mathrm{C}}$ (quadrada e retangular), como um tamanho ótimo de parcela (Xo) possível de ser utilizado em experimentos. A partir disso, foi calculada a diferença mínima significativa (d) entre as médias de tratamentos, pelo teste de Tukey, expressa em percentagem da média do experimento, em 448 cenários formados pela combinação de 16 tamanhos ótimos de parcela (Xo), i tratamentos $(\mathrm{i}=5,10,15 \mathrm{e}$ 20) e r repetições $(r=3,4,5,6,10,15$ e 20), em cada época de avaliação.

Para o cálculo da diferença mínima significativa (d), utilizou-se a expressão $\mathrm{d}=100\left(\mathrm{q}_{\alpha(\mathrm{i} ; \mathrm{GLE})} \sqrt{\mathrm{QME} / \mathrm{r}}\right) / \mathrm{m}$ em que: $\mathrm{q}_{\alpha(\mathrm{i} ; \mathrm{GLE})}$ é o valor crítico do teste de Tukey em nível $\alpha$ de probabilidade de erro $(\alpha=0,05$, nesse estudo); i é o número de tratamentos; GLE é o número de graus de liberdade do erro, ou seja, (i-1)(r-1) para o delineamento blocos ao acaso; QME é o quadrado médio do erro $\left(\mathrm{QME}=\mathrm{s}^{2}\right.$, nesse estudo); r é o número de repetições; e m é a média do experimento. Percentuais menores de $\mathrm{d}$ indicam maior precisão, ou seja, diferenças menores entre médias de tratamentos serão consideradas significativas, enquanto que percentuais maiores de $\mathrm{d}$ indicam menor precisão experimental. As análises estatísticas foram realizadas com auxílio do aplicativo Microsoft Office Excel ${ }^{\circledR}$ e do software $R$ (R DEVELOPMENT CORE TEAM, 2015).

\section{RESULTADOS E DISCUSSÃO}

O coeficiente de autocorrelação espacial de primeira ordem $(\rho)$ oscilou entre 0,32 e 0,59 , aos 123DAS (primeira época de avaliação), entre 0,24 e 0,59 , aos 137DAS (segunda época de avaliação) e entre 0,30 e 0,62, aos 150DAS (terceira época de avaliação) (Tabela 1). Essa reduzida oscilação de $\rho$ entre os 16 planos de UEB com tamanhos $\mathrm{X}=\mathrm{X}_{\mathrm{L}} \times \mathrm{X}_{\mathrm{C}}(\mathrm{X}=1,2$, $3,4,6,8,9,12,16 \mathrm{~m}^{2}$ ) e formas $\mathrm{X}_{\mathrm{L}} \times \mathrm{X}_{\mathrm{C}}$ (quadrada e retangular), evidencia possível independência do $\rho$ em relação aos tamanhos de UEB (Figura 1). O tamanho ótimo de parcela, calculado pela expressão $X_{0}=\left(10 \sqrt[3]{2\left(1-\rho^{2}\right) \mathrm{s}^{2} \mathrm{~m}}\right) / \mathrm{m}$ (PARANAÍBA et al., 2009), com valores fixos de variância $\left(\mathrm{s}^{2}\right)$ e média $(\mathrm{m})$, será máximo e mínimo quando houver, respectivamente, independência $(\rho=0)$ e dependência $(\rho=|1|)$ entre UEB adjacentes.

Nas três épocas de avaliação da massa de matéria verde de tremoço branco, houve padrão de aumento linear do desvio padrão (s) e da média (m), com o acréscimo dos tamanhos de UEB (X, em UEB) (Tabela 1 e Figura 1). O acréscimo do desvio padrão (s), em menor proporção que o aumento da média $(\mathrm{m})$, resultou em decréscimos do coeficiente de variação do ensaio $(\mathrm{CV}=100 \mathrm{~s} / \mathrm{m}$, em \%), com padrão de modelo potência. Portanto, apesar da reduzida oscilação de $\rho$, entre os 16 planos de UEB, é possível que o tamanho ótimo de parcela (Xo) seja influenciado pelo tamanho da UEB, devido à variação do desvio padrão (s) e da média (m).

Com o acréscimo dos tamanhos de UEB (X, em UEB), houve redução do tamanho ótimo de parcela (Xo, em UEB), com padrão de modelo potência (Figura 1), oscilando entre 6,90 e 4,28UEB, aos 123DAS, entre 5,87 e 3,61UEB, aos 137DAS e, entre 5,98 e 3,83UEB, aos 150DAS (Tabela 1). Já o tamanho ótimo de parcela $\left(\mathrm{Xo}, \mathrm{em}^{2}\right.$ ) aumentou, linearmente, com o acréscimo dos tamanhos de UEB (X, em UEB) (Figura 1), oscilando entre 6,90 e $68,53 \mathrm{~m}^{2}$, aos $123 \mathrm{DAS}$, entre 5,87 e $57,73 \mathrm{~m}^{2}$, aos 137DAS e, entre 5,98 e $61,28 \mathrm{~m}^{2}$, aos 150DAS (Tabela 1). Assim, pode-se inferir que o tamanho ótimo de parcela, para a avaliação da massa de matéria verde de tremoço branco, depende do tamanho da unidade experimental básica. Consequentemente, pode-se inferir que o tamanho da unidade experimental básica deve ser o menor possível, para não superestimar o tamanho ótimo de parcela.

$\mathrm{Na}$ prática, ao planejar o experimento a campo, o tamanho de parcela deve ser estabelecido em unidade de área, ou seja, em $\mathrm{m}^{2}$. Assim, devido à relação de dependência entre o tamanho ótimo de parcela e o tamanho da unidade experimental básica, o tamanho ótimo de parcela pode ser superestimado, caso a coleta de dados no ensaio de uniformidade não seja realizada em UEB de menor tamanho possível, compatível aos tratamentos a serem avaliados.

Com base na média das três épocas de avaliação, pode-se inferir que, para cada aumento de uma UEB $\left(1 \mathrm{~m}^{2}\right)$, houve acréscimo de $3,8034 \mathrm{~m}^{2}$ [média entre os coeficientes angulares do modelo linear das três avaliações, ou seja, $(4,1848+3,4444+3,7809) / 3=3,8034$ ] na estimativa do tamanho ótimo de parcela (Figura 1). 


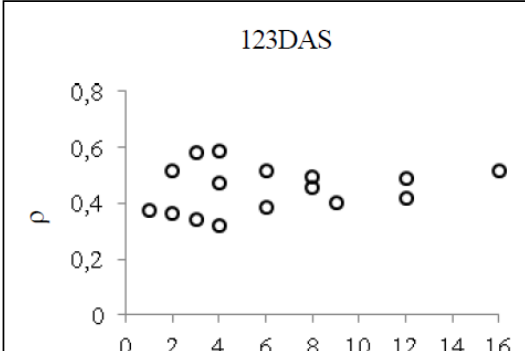

137DAS

150DAS
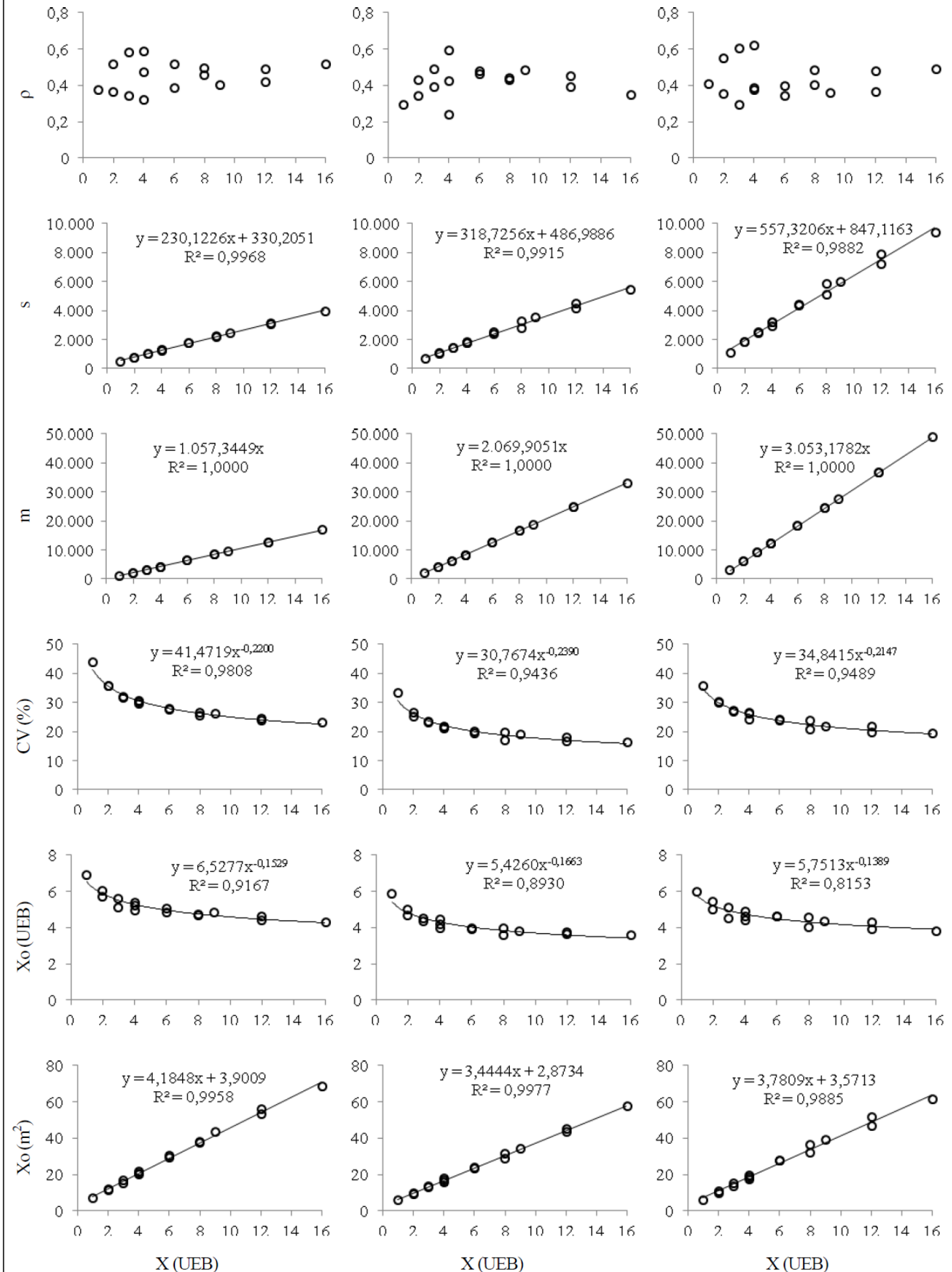

Figura 1 - Relações entre as variáveis dependentes - coeficiente de autocorrelação espacial de primeira ordem ( $\rho$ ), variância $\left(\mathrm{s}^{2}\right)$, desvio padrão $(\mathrm{s})$, média $(\mathrm{m})$, coeficiente de variação do ensaio (CV, em \%), tamanho ótimo de parcela (Xo, em UEB) e tamanho ótimo de parcela (Xo, em $\mathrm{m}^{2}$ ) - com a variável independente tamanho de UEB (X, em UEB), para a massa de matéria verde de tremoço branco (Lupinus albus L.), em gramas $\mathrm{m}^{-2}$, avaliada aos 123, 137 e 150 dias após a semeadura (DAS). 
Em pesquisa com produtividade de tubérculos de batata (Solanum tuberosum L.), OLIVEIRA et al. (2005) utilizaram o método da curvatura máxima modificada (MEIER \& LESSMAN, 1971) e a relação entre o tamanho ótimo de parcela (Xo, em $\mathrm{m}^{2}$ ) e o tamanho de UEB (X, em UEB) foi expressa por $\mathrm{Xo}=0,5287+0,3169 \mathrm{X}+0,0243 \mathrm{X}^{2}$ $\left(\mathrm{R}^{2}=0,9855\right)$. As distintas culturas, tamanhos de UEB planejados e métodos de estimação de Xo, podem explicar esses distintos padrões observados, ou seja, o linear desse estudo e o quadrático, em OLIVEIRA et al. (2005).

O fato de haver redução do coeficiente de variação $(\mathrm{CV})$ com o acréscimo dos tamanhos de UEB (X, em UEB) evidencia melhoria da precisão experimental, com parcelas maiores. No entanto, os modelos do tipo potência revelam que, a partir do plano com menor tamanho de UEB $\left(X=1 \times 1=1 \quad \mathrm{UEB}=1,00 \mathrm{~m}^{2}\right)$, houve ganhos expressivos em precisão (redução do $\mathrm{CV}$ ), com pequenos acréscimos no tamanho da UEB e tendência de estabilização desses ganhos com o aumento das dimensões da UEB (Figura 1). Assim, para avaliar a massa de matéria verde de tremoço branco, é importante determinar a precisão experimental, em cenários formados por combinações de tamanhos de parcela e de números de tratamentos e de repetições, a fim de definir o melhor plano experimental, conforme sugerido em CARGNELUTTI FILHO et al. (2014).

A estatística diferença mínima significativa pelo teste de Tukey, por considerar a média e os números de tratamentos e de repetições, foi considerada mais apropriada que o coeficiente de variação (CARGNELUTTI FILHO et al., 2014), para a avaliação da precisão experimental. Neste estudo, assumiu-se que cada plano de UEB de tamanho $\left(\mathrm{X}=\mathrm{X}_{\mathrm{L}} \times \mathrm{X}_{\mathrm{C}}\right)$ (Tabela 1) é um tamanho ótimo de parcela (Xo) possível de ser utilizado em experimentos. Assim, com as estimativas da variância $\left(\mathrm{s}^{2}\right)$ e da média $(\mathrm{m})$, foi calculada a diferença mínima significativa (d) entre as médias de tratamentos, pelo teste de Tukey, expressa em percentagem da média do experimento, em 448 cenários formados pela combinação de 16 tamanhos ótimos de parcela (Xo), i tratamentos $(\mathrm{i}=5,10,15 \mathrm{e}$ 20) e $\mathrm{r}$ repetições $(\mathrm{r}=3,4,5,6,10,15$ e 20$)$, em três épocas de avaliação (Tabelas 2 e 3 ).

Menores estimativas de d são almejadas, pois quanto menor a estimativa de d, mais preciso é o experimento, ou seja, menores diferenças entre médias de tratamentos são identificadas como significativas (LÚCIO et al.,
1999; STORCK et al., 2011; CARGNELUTTI FILHO et al., 2014). Para valores fixos de tamanho de parcela e número de tratamentos, a precisão experimental melhorou (menores valores de d) gradativamente com o aumento do número de repetições, independentemente da época de avaliação (Tabelas 2 e 3). Como exemplo, aos 123DAS, para $\mathrm{Xo}=1 \mathrm{~m}^{2}$ e $\mathrm{i}=5$, o d reduziu de $123,3 \%$ com $\mathrm{r}=3$, para $38,6 \%$ com $r=20$ (Tabela 2 ).

Nas três épocas de avaliação, para valores fixos de tamanho de parcela e número de repetições, as estimativas de $\mathrm{d}$ aumentaram com o acréscimo do número de tratamentos. Como exemplo, aos 123DAS, para $\mathrm{Xo}=1 \mathrm{~m}^{2}$ e $\mathrm{r}=3$, o d aumentou de $123,3 \%$ com $i=5$, para $135,7 \%$ com $i=20$ (Tabelas 2 e 3). Mantendo fixos os números de tratamentos e de repetições, verificou-se redução de $\mathrm{d}$, com o acréscimo do tamanho de parcela. Por exemplo, aos 123DAS, para $\mathrm{i}=5$ e $\mathrm{r}=3$, o d reduziu de $123,3 \%$ com $\mathrm{Xo}=1 \mathrm{~m}^{2}$, para $65,4 \%$ com $X o=16 \mathrm{~m}^{2}$ (Tabela 2 ).

As estimativas de d, para valores fixos de tamanho ótimo de parcela, número de tratamento e número de repetição, foram maiores aos 123DAS (menor precisão), menores aos 137DAS (maior precisão) e intermediários aos 150DAS. Por exemplo, para $\mathrm{Xo}=1 \mathrm{~m}^{2}, \mathrm{i}=5 \mathrm{e} \mathrm{r}=3$, o d foi de $123,3 \%, 93,7 \%$ e $101,1 \%$, para as avaliações aos 123, 137 e 150DAS, respectivamente (Tabela 2), o que evidencia precisão experimental diferenciada nessas épocas de avaliação.

Constatou-se que, para número fixo de tratamentos, parcelas menores com maior número de repetições, conferem maior precisão experimental (menores estimativas de d) (Tabelas 2 e 3). Por exemplo, aos 123DAS, para avaliar cinco tratamentos, precisão de $45 \%$, pode ser obtida usando parcelas de $1 \mathrm{~m}^{2}$ e 15 repetições $\left(15 \mathrm{~m}^{2}\right.$ por tratamento) ou parcelas de $9 \mathrm{~m}^{2} \mathrm{e}$ seis repetições $\left(54 \mathrm{~m}^{2}\right.$ por tratamento). Portanto, nesse cenário, com parcelas de $1 \mathrm{~m}^{2}$, embora o pesquisador possa ter maior dispêndio de mão-de-obra e mais custos financeiros para mensuração de determinada variável, pela necessidade de 15 repetições (15 avaliações), a redução da área necessária para o experimento seria um aspecto vantajoso a ser considerado no planejamento experimental. Inúmeros outros exemplos, poderiam ser formados (Tabelas 2 e 3 ) para a constatação de que, para uma mesma precisão, parcelas menores e maior número de repetições são mais eficientes no uso da área experimental, conforme já discutido nos estudos de OLIVEIRA et al. (2005), STORCK et al. (2011) e CARGNELUTTI FILHO et al. (2014).

Pode-se inferir que os cenários formados por combinações de tamanhos de parcela, números 
Tabela 2 - Diferença mínima significativa (d) entre as médias de tratamentos, pelo teste de Tukey, a 5\% de probabilidade, em percentagem da média do experimento, em 224 cenários $^{(1)}$, para avaliação da massa de matéria verde de tremoço branco (Lupinus albus L.), em delineamento blocos ao acaso. Para o cálculo de d, assumiu-se cada plano de UEB de tamanho $\left(\mathrm{X}=\mathrm{X}_{\mathrm{L}} \times \mathrm{X}_{\mathrm{C}}, \mathrm{em} \mathrm{m}^{2}\right)$ como um tamanho ótimo de parcela $\left(\mathrm{Xo}, \mathrm{em}^{2}\right.$ ) possível de ser utilizado em experimentos.

\begin{tabular}{|c|c|c|c|c|c|c|c|c|c|c|c|c|c|c|c|c|c|c|c|}
\hline \multirow{2}{*}{ DAS } & \multirow{2}{*}{ Plano } & \multirow{2}{*}{$\mathrm{X}_{\mathrm{L}}$} & \multirow{2}{*}{$\mathrm{X}_{\mathrm{C}}$} & \multirow{2}{*}{$X\left(\mathrm{~m}^{2}\right)$} & \multirow{2}{*}{$\mathrm{Xo}\left(\mathrm{m}^{2}\right)$} & \multicolumn{7}{|c|}{-----------------------5 tratamentos---------------------- } & \multicolumn{7}{|c|}{ 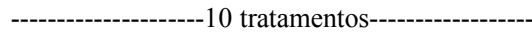 } \\
\hline & & & & & & 3 & 4 & 5 & 6 & 10 & 15 & 20 & 3 & 4 & 5 & 6 & 10 & 15 & 20 \\
\hline 123 & 1 & 1 & 1 & 1 & 1 & 123,3 & 98,5 & 84,7 & 75,5 & 56,1 & 45,0 & 38,6 & 128,0 & 106,3 & 93,2 & 84,0 & 63,6 & 51,4 & 44,3 \\
\hline 123 & 2 & 1 & 2 & 2 & 2 & 100,7 & 80,4 & 69,1 & 61,7 & 45,8 & 36,7 & 31,5 & 104,5 & 86,8 & 76,0 & 68,5 & 51,9 & 42,0 & 36,2 \\
\hline 123 & 3 & 2 & 1 & 2 & 2 & 100,5 & 80,3 & 69,1 & 61,6 & 45,8 & 36,7 & 31,5 & 104,3 & 86,7 & 75,9 & 68,5 & 51,9 & 41,9 & 36,1 \\
\hline 123 & 4 & 1 & 3 & 3 & 3 & 88,7 & 70,9 & 61,0 & 54,3 & 40,4 & 32,4 & 27,8 & 92,1 & 76,5 & 67,0 & 60,4 & 45,8 & 37,0 & 31,9 \\
\hline 123 & 5 & 3 & 1 & 3 & 3 & 90,4 & 72,2 & 62,1 & 55,4 & 41,2 & 33,0 & 28,3 & 93,8 & 78,0 & 68,3 & 61,6 & 46,6 & 37,7 & 32,5 \\
\hline 123 & 6 & 1 & 4 & 4 & 4 & 83,6 & 66,8 & 57,4 & 51,2 & 38,1 & 30,5 & 26,2 & 86,8 & 72,1 & 63,2 & 56,9 & 43,1 & 34,9 & 30,1 \\
\hline 123 & 7 & 2 & 2 & 4 & 4 & 85,1 & 68,0 & 58,4 & 52,1 & 38,7 & 31,0 & 26,6 & 88,3 & 73,3 & 64,3 & 57,9 & 43,9 & 35,5 & 30,6 \\
\hline 123 & 8 & 4 & 1 & 4 & 4 & 86,4 & 69,1 & 59,4 & 52,9 & 39,3 & 31,5 & 27,1 & 89,7 & 74,5 & 65,3 & 58,9 & 44,6 & 36,0 & 31,1 \\
\hline 123 & 9 & 2 & 3 & 6 & 6 & 77,6 & 62,0 & 53,3 & 47,5 & 35,3 & 28,3 & 24,3 & 80,5 & 66,9 & 58,6 & 52,8 & 40,0 & 32,3 & 27,9 \\
\hline 123 & 10 & 3 & 2 & 6 & 6 & 78,6 & 62,8 & 54,0 & 48,1 & 35,8 & 28,7 & 24,6 & 81,5 & 67,7 & 59,3 & 53,5 & 40,5 & 32,8 & 28,2 \\
\hline 123 & 11 & 2 & 4 & 8 & 8 & 72,4 & 57,8 & 49,7 & 44,3 & 32,9 & 26,4 & 22,7 & 75,1 & 62,4 & 54,7 & 49,3 & 37,3 & 30,2 & 26,0 \\
\hline 123 & 12 & 4 & 2 & 8 & 8 & 75,1 & 60,0 & 51,6 & 46,0 & 34,2 & 27,4 & 23,5 & 77,9 & 64,7 & 56,7 & 51,1 & 38,7 & 31,3 & 27,0 \\
\hline 123 & 13 & 3 & 3 & 9 & 9 & 73,5 & 58,8 & 50,5 & 45,0 & 33,5 & 26,8 & 23,0 & 76,3 & 63,4 & 55,5 & 50,1 & 37,9 & 30,7 & 26,4 \\
\hline 123 & 14 & 3 & 4 & 12 & 12 & 67,3 & 53,8 & 46,2 & 41,2 & 30,6 & 24,6 & 21,1 & 69,9 & 58,0 & 50,8 & 45,8 & 34,7 & 28,1 & 24,2 \\
\hline 123 & 15 & 4 & 3 & 12 & 12 & 69,5 & 55,5 & 47,7 & 42,6 & 31,6 & 25,3 & 21,8 & 72,1 & 59,9 & 52,5 & 47,3 & 35,8 & 29,0 & 25,0 \\
\hline 123 & 16 & 4 & 4 & 16 & 16 & 65,4 & 52,2 & 44,9 & 40,0 & 29,7 & 23,8 & 20,5 & 67,8 & 56,4 & 49,4 & 44,5 & 33,7 & 27,3 & 23,5 \\
\hline 137 & 1 & 1 & 1 & 1 & 1 & 93,7 & 74,9 & 64,4 & 57,4 & 42,7 & 34,2 & 29,4 & 97,3 & 80,8 & 70,8 & 63,8 & 48,4 & 39,1 & 33,7 \\
\hline 137 & 2 & 1 & 2 & 2 & 2 & 74,9 & 59,8 & 51,4 & 45,9 & 34,1 & 27,3 & 23,5 & 77,7 & 64,6 & 56,6 & 51,0 & 38,6 & 31,2 & 26,9 \\
\hline 137 & 3 & 2 & 1 & 2 & 2 & 71,1 & 56,8 & 48,9 & 43,6 & 32,4 & 26,0 & 22,3 & 73,8 & 61,3 & 53,7 & 48,4 & 36,7 & 29,7 & 25,6 \\
\hline 137 & 4 & 1 & 3 & 3 & 3 & 65,5 & 52,4 & 45,0 & 40,1 & 29,8 & 23,9 & 20,5 & 68,0 & 56,5 & 49,5 & 44,6 & 33,8 & 27,3 & 23,6 \\
\hline 137 & 5 & 3 & 1 & 3 & 3 & 65,9 & 52,7 & 45,3 & 40,4 & 30,0 & 24,1 & 20,7 & 68,4 & 56,9 & 49,8 & 44,9 & 34,0 & 27,5 & 23,7 \\
\hline 137 & 6 & 1 & 4 & 4 & 4 & 60,7 & 48,5 & 41,7 & 37,2 & 27,6 & 22,2 & 19,0 & 63,0 & 52,4 & 45,9 & 41,4 & 31,3 & 25,3 & 21,8 \\
\hline 137 & 7 & 2 & 2 & 4 & 4 & 59,8 & 47,8 & 41,1 & 36,6 & 27,2 & 21,8 & 18,7 & 62,1 & 51,6 & 45,2 & 40,7 & 30,8 & 24,9 & 21,5 \\
\hline 137 & 8 & 4 & 1 & 4 & 4 & 6 & 49,3 & 42,4 & 37,8 & 28,1 & 22,5 & 19,3 & & 53,2 & 46,6 & 42,0 & 31,8 & 25,7 & 2,2 \\
\hline 137 & 9 & 2 & 3 & 6 & 6 & 54,7 & 43,7 & 37,6 & 33,5 & 24,9 & 20,0 & 17,1 & 56,8 & 47,2 & 41,3 & 37,2 & 28,2 & 22,8 & 19,7 \\
\hline 137 & 10 & 3 & 2 & 6 & 6 & 56,9 & 45,4 & 39,1 & 34,8 & 25,9 & 20,7 & 17,8 & 59,0 & 49,0 & 42,9 & 38,7 & 29,3 & 23,7 & 20,4 \\
\hline 137 & 11 & 2 & 4 & 8 & 8 & 47,9 & 38,3 & 32,9 & 29,4 & 21,8 & 17,5 & 15,0 & 49,8 & 41,3 & 36,2 & 32,6 & 24,7 & 20,0 & 17,2 \\
\hline 137 & 12 & 4 & 2 & 8 & 8 & 55,2 & 44,1 & 37,9 & 33,8 & 25,1 & 20,2 & 17,3 & 57,3 & 47,6 & 41,7 & 37,6 & 28,5 & 23,0 & 19,8 \\
\hline 137 & 13 & 3 & 3 & 9 & 9 & 53,2 & 42,5 & 36,6 & 32,6 & 24,2 & 19,4 & 16,7 & 55,2 & 45,9 & 40,2 & 36,2 & 27,5 & 22,2 & 19,1 \\
\hline 137 & 14 & 3 & 4 & 12 & 12 & 47,4 & 37,9 & 32,6 & 29,1 & 21,6 & 17,3 & 14,9 & 49,2 & 40,9 & 35,8 & 32,3 & 24,5 & 19,8 & 17,1 \\
\hline 137 & 15 & 4 & 3 & 12 & 12 & 51,2 & 40,9 & 35,2 & 31,4 & 23,3 & 18,7 & 16,0 & 53,2 & 44,2 & 38,7 & 34,9 & 26,4 & 21,4 & 18,4 \\
\hline 137 & 16 & 4 & 4 & 16 & 16 & 46,1 & 36,9 & 31,7 & 28,3 & 21,0 & 16,8 & 14,5 & 47,9 & 39,8 & 34,8 & 31,4 & 23,8 & 19,2 & 16,6 \\
\hline 150 & 1 & 1 & 1 & 1 & 1 & 101,1 & 80,8 & 69,4 & 61,9 & 46,0 & 36,9 & 31,7 & 104,9 & 87,2 & 76,4 & 68,8 & 52,1 & 42,2 & 36,3 \\
\hline 150 & 2 & 1 & 2 & 2 & 2 & 85,1 & 68,0 & 58,5 & 52,2 & 38,8 & 31,1 & 26,7 & 88,4 & 73,4 & 64,3 & 58,0 & 43,9 & 35,5 & 30,6 \\
\hline 150 & 3 & 2 & 1 & 2 & 2 & 84,0 & 67,1 & 57,7 & 51,4 & 38,2 & 30,6 & 26,3 & 87,2 & 72,4 & 63,4 & 57,2 & 43,3 & 35,0 & 30,2 \\
\hline 150 & 4 & 1 & 3 & 3 & 3 & 76,8 & 61,3 & 52,7 & 47,0 & 34,9 & 28,0 & 24,1 & 79,7 & 66,2 & 58,0 & 52,3 & 39,6 & 32,0 & 27,6 \\
\hline 150 & 5 & 3 & 1 & 3 & 3 & 76,2 & 60,8 & 52,3 & 46,6 & 34,7 & 27,8 & 23,9 & 79,0 & 65,7 & 57,5 & 51,9 & 39,3 & 31,8 & 27,4 \\
\hline 150 & 6 & 1 & 4 & 4 & 4 & 68,0 & 54,4 & 46,7 & 41,7 & 31,0 & 24,8 & 21,3 & 70,6 & 58,7 & 51,4 & 46,3 & 35,1 & 28,4 & 24,5 \\
\hline 150 & 7 & 2 & 2 & 4 & 4 & 73,9 & 59,1 & 50,8 & 45,3 & 33,6 & 27,0 & 23,2 & 76,7 & 63,7 & 55,8 & 50,3 & 38,1 & 30,8 & 26,6 \\
\hline 150 & 8 & 4 & 1 & 4 & 4 & 74,5 & 59,5 & 51,2 & 45,6 & 33,9 & 27,2 & 23,3 & 77,3 & 64,3 & 56,3 & 50,7 & 38,4 & 31,1 & 26,8 \\
\hline 150 & 9 & 2 & 3 & 6 & 6 & 67,1 & 53,6 & 46,1 & 41,1 & 30,6 & 24,5 & 21,0 & 69,7 & 57,9 & 50,7 & 45,7 & 34,6 & 28,0 & 24,1 \\
\hline 150 & 10 & 3 & 2 & 6 & 6 & 68,4 & 54,7 & 47,0 & 41,9 & 31,1 & 25,0 & 21,4 & 71,0 & 59,0 & 51,7 & 46,6 & 35,3 & 28,5 & 24,6 \\
\hline 150 & 11 & 2 & 4 & 8 & 8 & 58,6 & 46,8 & 40,3 & 35,9 & 26,7 & 21,4 & 18,4 & 60,8 & 50,5 & 44,3 & 39,9 & 30,2 & 24,4 & 21,1 \\
\hline 150 & 12 & 4 & 2 & 8 & 8 & 67,2 & 53,7 & 46,1 & 41,1 & 30,6 & 24,5 & 21,0 & 69,7 & 57,9 & 50,7 & 45,7 & 34,7 & 28,0 & 24,1 \\
\hline 150 & 13 & 3 & 3 & 9 & 9 & 61,7 & 49,3 & 42,4 & 37,8 & 28,1 & 22,5 & 19,3 & 64,0 & 53,2 & 46,6 & 42,0 & 31,8 & 25,7 & 22,2 \\
\hline 150 & 14 & 3 & 4 & 12 & 12 & 55,3 & 44,2 & 38,0 & 33,9 & 25,2 & 20,2 & 17,3 & 57,4 & 47,7 & 41,8 & 37,6 & 28,5 & 23,0 & 19,9 \\
\hline 150 & 15 & 4 & 3 & 12 & 12 & 60,9 & 48,7 & 41,8 & 37,3 & 27,7 & 22,2 & 19,1 & 63,2 & 52,5 & 46,0 & 41,5 & 31,4 & 25,4 & 21,9 \\
\hline 150 & 16 & 4 & 4 & 16 & 16 & 54,3 & 43,4 & 37,3 & 33,2 & 24,7 & 19,8 & 17,0 & 56,3 & 46,8 & 41,0 & 37,0 & 28,0 & 22,6 & 19,5 \\
\hline
\end{tabular}

${ }^{(1)}$ Cenários formados pelas combinações de 16 tamanhos ótimos de parcela $(X o$, em m²), i tratamentos $(i=5$ e 10$)$ e $r$ repetições $(r=3,4,5,6$, 10,15 e 20). $\mathrm{X}_{\mathrm{L}}$ : UEB adjacentes à linha; $\mathrm{X}_{\mathrm{C}}$ : UEB adjacentes à coluna.

de tratamentos e de repetições e épocas de avaliação geraram distintas precisões experimentais. Portanto, ao planejar o experimento, para determinada precisão experimental, é importante considerar, conjuntamente, esses fatores, além de possíveis limitações de área experimental e custos financeiros para as avaliações. Na prática, os resultados deste estudo possibilitam ao pesquisador definir, adequadamente, o tamanho de parcela e o número de repetições a ser utilizado na avaliação de determinado número de tratamentos e 
Tabela 3 - Diferença mínima significativa (d) entre as médias de tratamentos, pelo teste de Tukey, a 5\% de probabilidade, em percentagem da média do experimento, em 224 cenários $^{(1)}$, para avaliação da massa de matéria verde de tremoço branco (Lupinus albus L.), em delineamento blocos ao acaso. Para o cálculo de d, assumiu-se cada plano de UEB de tamanho $\left(\mathrm{X}=\mathrm{X}_{\mathrm{L}} \times \mathrm{X}_{\mathrm{C}}, \mathrm{em}^{2}\right)$ como um tamanho ótimo de parcela $\left(\mathrm{Xo}, \mathrm{em} \mathrm{m}^{2}\right.$ ) possível de ser utilizado em experimentos.

\begin{tabular}{|c|c|c|c|c|c|c|c|c|c|c|c|c|c|c|c|c|c|c|c|}
\hline \multirow{2}{*}{ DAS } & \multirow{2}{*}{ Plano } & \multirow{2}{*}{$\mathrm{X}_{\mathrm{L}}$} & \multirow{2}{*}{$\mathrm{X}_{\mathrm{C}}$} & \multirow{2}{*}{$X\left(m^{2}\right)$} & \multirow{2}{*}{$\mathrm{Xo}\left(\mathrm{m}^{2}\right)$} & \multirow[b]{2}{*}{3} & \multirow[b]{2}{*}{4} & \multirow[b]{2}{*}{5} & \multirow[b]{2}{*}{6} & \multirow[b]{2}{*}{10} & \multirow[b]{2}{*}{15} & \multirow[b]{2}{*}{20} & \multirow[b]{2}{*}{3} & & & 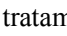 & ntos & & \\
\hline & & & & & & & & & & & & & & 4 & 5 & 6 & 10 & 15 & 20 \\
\hline 123 & 1 & 1 & 1 & 1 & 1 & 132,3 & 111,3 & 98,1 & 88,7 & 67,6 & 54,8 & 47,3 & 135,7 & 114,8 & 101,5 & 92,0 & 70,4 & 57,2 & 49,4 \\
\hline 123 & 2 & 1 & 2 & 2 & 2 & 108,0 & 90,8 & 80,0 & 72,4 & 55,2 & 44,8 & 38,6 & 110,8 & 93,7 & 82,9 & 75,1 & 57,5 & 46,7 & 40,3 \\
\hline 123 & 3 & 2 & 1 & 2 & 2 & 107,9 & 90,7 & 79,9 & 72,3 & 55,1 & 44,7 & 38,6 & 110,6 & 93,6 & 82,8 & 75,0 & 57,4 & 46,6 & 40,2 \\
\hline 123 & 4 & 1 & 3 & 3 & 3 & 95,2 & 80,1 & 70,6 & 63,8 & 48,7 & 39,5 & 34,1 & 97,6 & 82,6 & 73,1 & 66,2 & 50,7 & 41,1 & 35,5 \\
\hline 123 & 5 & 3 & 1 & 3 & 3 & 97,0 & 81,6 & 71,9 & 65,1 & 49,6 & 40,2 & 34,7 & 99,5 & 84,2 & 74,4 & 67,5 & 51,6 & 41,9 & 36,2 \\
\hline 123 & 6 & 1 & 4 & 4 & 4 & 89,7 & 75,5 & 66,5 & 60,2 & 45,9 & 37,2 & 32,1 & 92,0 & 77,9 & 68,8 & 62,4 & 47,7 & 38,8 & 33,5 \\
\hline 123 & 7 & 2 & 2 & 4 & 4 & 91,3 & 76,8 & 67,6 & 61,2 & 46,7 & 37,8 & 32,6 & 93,6 & 79,2 & 70,0 & 63,5 & 48,6 & 39,4 & 34,1 \\
\hline 123 & 8 & 4 & 1 & 4 & 4 & 92,7 & 78,0 & 68,7 & 62,2 & 47,4 & 38,4 & 33,2 & 95,1 & 80,5 & 71,2 & 64,5 & 49,3 & 40,1 & 34,6 \\
\hline 123 & 9 & 2 & 3 & 6 & 6 & 83,2 & 70,0 & 61,7 & 55,8 & 42,5 & 34,5 & 29,8 & 85,3 & 72,2 & 63,9 & 57,9 & 44,3 & 35,9 & 31,0 \\
\hline 123 & 10 & 3 & 2 & 6 & 6 & 84,3 & 70,9 & 62,5 & 56,5 & 43,1 & 34,9 & 30,2 & 86,4 & 73,2 & 64,7 & 58,6 & 44,8 & 36,4 & 31,4 \\
\hline 123 & 11 & 2 & 4 & 8 & 8 & 77,6 & 65,3 & 57,5 & 52,1 & 39,7 & 32,2 & 27,8 & 79,6 & 67,4 & 59,6 & 54,0 & 41,3 & 33,5 & 29,0 \\
\hline 123 & 12 & 4 & 2 & 8 & 8 & 80,5 & 67,7 & 59,7 & 54,0 & 41,2 & 33,4 & 28,8 & 82,6 & 69,9 & 61,8 & 56,0 & 42,8 & 34,8 & 30,0 \\
\hline 123 & 13 & 3 & 3 & 9 & 9 & 78,9 & 66,4 & 58,5 & 52,9 & 40,3 & 32,7 & 28,2 & 80,9 & 68,5 & 60,5 & 54,9 & 42,0 & 34,1 & 29,4 \\
\hline 123 & 14 & 3 & 4 & 12 & 12 & 72,2 & 60,7 & 53,5 & 48,4 & 36,9 & 29,9 & 25,8 & 74,1 & 62,7 & 55,4 & 50,2 & 38,4 & 31,2 & 26,9 \\
\hline 123 & 15 & 4 & 3 & 12 & 12 & 74,5 & 62,7 & 55,2 & 50,0 & 38,1 & 30,9 & 26,7 & 76,4 & 64,7 & 57,2 & 51,8 & 39,7 & 32,2 & 27,8 \\
\hline 123 & 16 & 4 & 4 & 16 & 16 & 70,1 & 59,0 & 52,0 & 47,0 & 35,8 & 29,1 & 25,1 & 71,9 & 60,9 & 53,8 & 48,8 & 37,3 & 30,3 & 26,2 \\
\hline 137 & 1 & 1 & 1 & 1 & 1 & 100,6 & 84,6 & 74,5 & 67,4 & 51,4 & 41,7 & 36,0 & 103,1 & 87,3 & 77,2 & 70,0 & 53,5 & 43,4 & 37,5 \\
\hline 137 & 2 & 1 & 2 & 2 & 2 & 80,3 & 67,6 & 59,5 & 53,9 & 41,1 & 33,3 & 28,7 & 82,4 & 69,7 & 61,6 & 55,9 & 42,7 & 34,7 & 30,0 \\
\hline 137 & 3 & 2 & 1 & 2 & 2 & 76,3 & 64,2 & 56,6 & 51,2 & 39,0 & 31,6 & 27,3 & 78,3 & 66,2 & 58,6 & 53,1 & 40,6 & 33,0 & 28,5 \\
\hline 137 & 4 & 1 & 3 & 3 & 3 & 70,3 & 59,1 & 52,1 & 47,2 & 36,0 & 29,1 & 25,2 & 72,1 & 61,0 & 54,0 & 48,9 & 37,4 & 30,4 & 26,2 \\
\hline 137 & 5 & 3 & 1 & 3 & 3 & 70,7 & 59,5 & 52,4 & 47,4 & 36,2 & 29,3 & 25,3 & 72,6 & 61,4 & 54,3 & 49,2 & 37,6 & 30,6 & 26,4 \\
\hline 137 & 6 & 1 & 4 & 4 & 4 & 65,2 & 54,8 & 48,3 & 43,7 & 33,3 & 27,0 & 23,3 & 66,8 & 56,6 & 50,0 & 45,3 & 34,7 & 28,1 & 24,3 \\
\hline 137 & 7 & 2 & 2 & 4 & 4 & 64,1 & 54,0 & 47,5 & 43,0 & 32,8 & 26,6 & 22,9 & 65,8 & 55,7 & 49,2 & 44,6 & 34,1 & 27,7 & 23,9 \\
\hline 137 & 8 & 4 & 1 & 4 & 4 & 66,2 & 55,7 & 49,1 & 44,4 & 33,9 & 27,5 & 23,7 & 67,9 & 57,5 & 50,8 & 46,1 & 35,2 & 28,6 & 24,7 \\
\hline 137 & 9 & 2 & 3 & 6 & 6 & 58,7 & 49,4 & 43,5 & 39,4 & 30,0 & 24,3 & 21,0 & 60,2 & 50,9 & 45,0 & 40,8 & 31,2 & 25,3 & 21,9 \\
\hline 137 & 10 & 3 & 2 & 6 & 6 & 61,0 & 51,3 & 45,2 & 40,9 & 31,2 & 25,3 & 21,8 & 62,6 & 52,9 & 46,8 & 42,4 & 32,5 & 26,3 & 22,8 \\
\hline 137 & 11 & 2 & 4 & 8 & 8 & 51,4 & 43,3 & 38,1 & 34,5 & 26,3 & 21,3 & 18,4 & 52,7 & 44,6 & 39,5 & 35,8 & 27,4 & 22,2 & 19,2 \\
\hline 137 & 12 & 4 & 2 & 8 & 8 & 59,3 & 49,8 & 43,9 & 39,7 & 30,3 & 24,6 & 21,2 & 60,8 & 51,4 & 45,5 & 41,2 & 31,5 & 25,6 & 22,1 \\
\hline 137 & 13 & 3 & 3 & 9 & 9 & 57,1 & 48,0 & 42,3 & 38,3 & 29,2 & 23,7 & 20,4 & 58,6 & 49,6 & 43,8 & 39,7 & 30,4 & 24,7 & 21,3 \\
\hline 137 & 14 & 3 & 4 & 12 & 12 & 50,9 & 42,8 & 37,7 & 34,1 & 26,0 & 21,1 & 18,2 & 52,2 & 44,2 & 39,1 & 35,4 & 27,1 & 22,0 & 19,0 \\
\hline 137 & 15 & 4 & 3 & 12 & 12 & 55,0 & 46,2 & 40,7 & 36,9 & 28,1 & 22,8 & 19,7 & 56,4 & 47,7 & 42,2 & 38,2 & 29,2 & 23,7 & 20,5 \\
\hline 137 & 16 & 4 & 4 & 16 & 16 & 49,5 & 41,6 & 36,7 & 33,2 & 25,3 & 20,5 & 17,7 & 50,8 & 43,0 & 38,0 & 34,4 & 26,3 & 21,4 & 18,5 \\
\hline 150 & 1 & 1 & 1 & 1 & 1 & 108,5 & 91,2 & 80,4 & 72,7 & 55,5 & 45,0 & 38,8 & 111,2 & 94,1 & 83,2 & 75,4 & 57,7 & 46,9 & 40,5 \\
\hline 150 & 2 & 1 & 2 & 2 & 2 & 91,4 & 76,8 & 67,7 & 61,3 & 46,7 & 37,9 & 32,7 & 93,7 & 79,3 & 70,1 & 63,5 & 48,6 & 39,5 & 34,1 \\
\hline 150 & 3 & 2 & 1 & 2 & 2 & 90,1 & 75,8 & 66,8 & 60,4 & 46,1 & 37,3 & 32,2 & 92,4 & 78,2 & 69,1 & 62,7 & 47,9 & 38,9 & 33,6 \\
\hline 150 & 4 & 1 & 3 & 3 & 3 & 82,4 & 69,3 & 61,1 & 55,2 & 42,1 & 34,1 & 29,5 & 84,5 & 71,5 & 63,2 & 57,3 & 43,8 & 35,6 & 30,7 \\
\hline 150 & 5 & 3 & 1 & 3 & 3 & 81,7 & 68,7 & 60,6 & 54,8 & 41,8 & 33,9 & 29,2 & 83,8 & 70,9 & 62,7 & 56,8 & 43,5 & 35,3 & 30,5 \\
\hline 150 & 6 & 1 & 4 & 4 & 4 & 73,0 & 61,4 & 54,1 & 49,0 & 37,3 & 30,3 & 26,1 & 74,9 & 63,4 & 56,0 & 50,8 & 38,8 & 31,5 & 27,2 \\
\hline 150 & 7 & 2 & 2 & 4 & 4 & 79,3 & 66,7 & 58,8 & 53,2 & 40,5 & 32,9 & 28,4 & 81,3 & 68,8 & 60,8 & 55,2 & 42,2 & 34,3 & 29,6 \\
\hline 150 & 8 & 4 & 1 & 4 & 4 & 79,9 & 67,2 & 59,3 & 53,6 & 40,9 & 33,1 & 28,6 & 82,0 & 69,4 & 61,3 & 55,6 & 42,5 & 34,5 & 29,8 \\
\hline 150 & 9 & 2 & 3 & 6 & 6 & 72,0 & 60,6 & 53,4 & 48,3 & 36,8 & 29,8 & 25,8 & 73,9 & 62,5 & 55,3 & 50,1 & 38,3 & 31,1 & 26,9 \\
\hline 150 & 10 & 3 & 2 & 6 & 6 & 73,4 & 61,8 & 54,4 & 49,2 & 37,5 & 30,4 & 26,3 & 75,3 & 63,7 & 56,3 & 51,1 & 39,1 & 31,7 & 27,4 \\
\hline 150 & 11 & 2 & 4 & 8 & 8 & 62,9 & 52,9 & 46,6 & 42,2 & 32,1 & 26,1 & 22,5 & 64,5 & 54,6 & 48,2 & 43,7 & 33,5 & 27,2 & 23,5 \\
\hline 150 & 12 & 4 & 2 & 8 & 8 & 72,1 & 60,6 & 53,4 & 48,3 & 36,8 & 29,9 & 25,8 & 73,9 & 62,6 & 55,3 & 50,1 & 38,3 & 31,1 & 26,9 \\
\hline 150 & 13 & 3 & 3 & 9 & 9 & 66,2 & 55,6 & 49,0 & 44,4 & 33,8 & 27,4 & 23,7 & 67,9 & 57,4 & 50,8 & 46,0 & 35,2 & 28,6 & 24,7 \\
\hline 150 & 14 & 3 & 4 & 12 & 12 & 59,3 & 49,9 & 44,0 & 39,8 & 30,3 & 24,6 & 21,2 & 60,8 & 51,5 & 45,5 & 41,3 & 31,6 & 25,6 & 22,1 \\
\hline 150 & 15 & 4 & 3 & 12 & 12 & 65,3 & 55,0 & 48,4 & 43,8 & 33,4 & 27,1 & 23,4 & 67,0 & 56,7 & 50,1 & 45,4 & 34,8 & 28,2 & 24,4 \\
\hline 150 & 16 & 4 & 4 & 16 & 16 & 58,2 & 49,0 & 43,2 & 39,1 & 29,8 & 24,1 & 20,8 & 59,7 & 50,5 & 44,7 & 40,5 & 31,0 & 25,2 & 21,7 \\
\hline
\end{tabular}

${ }^{(1)}$ Cenários formados pelas combinações de 16 tamanhos ótimos de parcela $\left(\mathrm{Xo}, \mathrm{em} \mathrm{m}{ }^{2}\right)$, i tratamentos $(\mathrm{i}=15$ e 20$)$ e $\mathrm{r}$ repetições $(\mathrm{r}=3,4,5,6$, 10,15 e 20). $\mathrm{X}_{\mathrm{L}}$ : UEB adjacentes à linha; $\mathrm{X}_{\mathrm{C}}$ : UEB adjacentes à coluna.

com a precisão requerida (valor de d), e ainda que se ajuste a área experimental disponível. Além disso, as estimativas de variância $\left(\mathrm{s}^{2}\right)$ e média $(\mathrm{m})$ podem ser utilizadas para simular planejamentos experimentais para outros delineamentos experimentais e ainda com outros números de tratamentos e de repetições. Portanto, a definição do tamanho de parcela e do número de repetições, para o planejamento de experimentos com a cultura de tremoço branco, fica a critério do pesquisador que obtiver essas informações. 


\section{CONCLUSÃO}

A estimativa do tamanho ótimo de parcela, para a avaliação da massa de matéria verde de tremoço branco (Lupinus albus L.), depende do tamanho da unidade experimental básica. É indicado avaliar a massa de matéria verde em unidades experimentais básicas de menor tamanho possível, para serem usadas na estimação do tamanho ótimo de parcela.

\section{AGRADECIMENTOS}

Ao Conselho Nacional de Desenvolvimento Científico e Tecnológico (CNPq) e à Coordenação de Aperfeiçoamento de Pessoal de Nível Superior (CAPES), pela concessão de bolsas aos autores. À Fundação de Amparo à Pesquisa do Estado do Rio Grande do Sul (FAPERGS) pelo auxílio financeiro.

\section{REFERÊNCIAS}

CARGNELUTTI FILHO, A. et al. Tamanho de parcela para avaliar caracteres de tremoço branco em épocas de avaliação. Ciência Rural, v.45, p.206-214, 2015. Disponível em: $<$ http://www.scielo. br/pdf/cr/v45n2/0103-8478-cr-45-02-00206.pdf >. Acesso em: 25 maio 2015. doi: 10.1590/0103-8478cr20131255.

CARGNELUTTI FILHO, A. et al. Precisão experimental relacionada a tamanhos de parcelas, números de tratamentos e repetições em nabo forrageiro. Pesquisa Agropecuária Brasileira, v.49, p.428-439, 2014. Disponível em: <http://www.scielo.br/pdf/ pab/v49n6/0100-204X-pab-49-06-00428.pdf>. Acesso em: 25 maio 2015. doi: 10.1590/S0100-204X2013000700007.

HELDWEIN, A.B. et al. O clima de Santa Maria. Ciência \& Ambiente, v.38, p.43-58, 2009.

LÚCIO, A.D. et al. Classificação dos experimentos de competição de cultivares quanto a sua precisão. Pesquisa Agropecuária Gaúcha, v.5, p.99-103, 1999. Disponível em: $<$ http://www.fepagro.rs.gov.br/upload/1398909669_art_12. pdf $>$. Acesso em: 25 maio 2015.
MEIER, V.D.; LESSMAN, K.J. Estimation of optimum field plot shape and size for testing yield in Crambe abyssinica Hochst. Crop Science, v.11, p.648-650, 1971.

OLIVEIRA, S.J.R. et al. Plot size and experimental unit relationship in exploratory experiments. Scientia Agricola, v.62, p.585-589, 2005. Disponível em: <http://www.scielo.br/pdf/sa/ v62n6/a12v62n6.pdf>. Acesso em: 25 maio 2015. doi: 10.1590/ S0103-90162005000600012.

PARANAÍBA, P.F. et al. Tamanho ótimo de parcelas experimentais: proposição de métodos de estimação. Revista Brasileira de Biometria, v.27, p.255-268, 2009. Disponível em: $<$ http://jaguar. fcav.unesp.br/RME/fasciculos/v27/v27_n2/Patricia.pdf $>$. Acesso em: 25 maio 2015.

PEREIRA, G.A.M. et al. Fitomassa de adubos verdes e cobertura do solo na região do Alto Vale do Jequitinhonha, Minas Gerais. Revista Agro@mbiente On-line, v.6, p.110-116, 2012. Disponível em: <http://revista.ufrr.br/index.php/agroambiente/ article/view/696/777>. Acesso em: 25 maio 2015.

POTT, C.A. et al. Adubação verde como alternativa agroecológica para recuperação da fertilidade do solo. Ambiência - Revista do Setor de Ciências Agrárias e Ambientais, v.3, p.51-63, 2007. Disponível em: $<$ http://revistas.unicentro.br/index.php/ambiencia/ article/view/300/411>. Acesso em: 25 maio 2015.

R DEVELOPMENT CORE TEAM. R: a language and environment for statistical computing. Vienna: R Foundation for Statistical Computing, 2015. Disponível em: <http://www.R-project.org>. Acesso em: 25 maio 2015.

SANTOS, H.G. et al. Sistema brasileiro de classificação de solos. 2.ed. Rio de Janeiro: Embrapa Solos, 2006. 306p.

SOUZA, J.L. de; GUIMARÃES, G.P. Rendimento de massa de adubos verdes e o impacto na fertilidade do solo em sucessão de cultivos orgânicos. Bioscience Journal, v.29, p.1796-1805, 2013. Disponível em: <http://www.seer.ufu.br/index.php/biosciencejournal/ article/viewFile/21931/13417>. Acesso em: 25 maio 2015.

STORCK, L. et al. Experimentação vegetal. 3.ed. Santa Maria: UFSM, 2011. 200p. 